\title{
Ultrafast polarization switching in ferroelectrics
}

\author{
V. I. Yukalov $\circledast^{1,2}$ and E. P. Yukalova ${ }^{3}$ \\ ${ }^{1}$ Bogolubov Laboratory of Theoretical Physics, Joint Institute for Nuclear Research, Dubna 141980, Russia \\ ${ }^{2}$ Instituto de Fisica de São Carlos, Universidade de São Paulo, CP 369, São Carlos 13560-970, São Paulo, Brazil \\ ${ }^{3}$ Laboratory of Information Technologies, Joint Institute for Nuclear Research, Dubna 141980, Russia
}

(Received 15 September 2019; revised manuscript received 23 October 2019; published 27 November 2019)

\begin{abstract}
A method of ultrafast switching of ferroelectric polarization is suggested. The method is based on the interaction of a ferroelectric sample with the feedback field of a resonator in which the sample is inserted. The polarization reversal time can be of order of femtoseconds. The polarization switching produces a coherent electromagnetic pulse.
\end{abstract}

DOI: 10.1103/PhysRevResearch.1.033136

\section{INTRODUCTION}

Ferroelectric materials, possessing spontaneous electric polarization, can be harnessed for various electronic devices $[1,2]$. For example, they are used in devices regulating tunneling resistance [2] and enabling nonvolatile memory [3], in memristors [4], in neuromorphic networks [5,6], and in solar cells $[7,8]$.

To regulate the processing of such devices, it is often necessary to be able to quickly vary the direction and magnitude of ferroelectric polarization. There exist two ways of polarization switching that can be called inhomogeneous (or incoherent) and homogeneous (or coherent).

First, the inhomogeneous way of polarization switching was studied, being realized through the nucleation and growth of domains of opposite polarization, with moving domain walls under the influence of static electric fields [9-13]. This way, however, provides rather long switching times, of order of nanoseconds, being limited by the domain recrystallization time that is typically hundreds of picoseconds $[1,2,11,14,15]$. Similar slow switching in the nanoscale volume of a ferroelectric can be realized by mechanical deformation of a ferroelectric sample [16]. Another slow mechanism of polarization switching is due to the chemical oxidization at the surface of a ferroelectric film [17]. Because of the principal restriction of the inhomogeneous switching caused by the limited domain recrystallization time, it became necessary to find other ways that could provide much faster switching.

The other way that has been developed is the homogeneous (or coherent) switching process realized by external alternating fields, applied perpendicular to the ferroelectric polarization, in the optical [18-21], terahertz [22-28], or infrared [29] regions. Under this process, the alternating field acts directly on all ions of a single-domain sample, and

Published by the American Physical Society under the terms of the Creative Commons Attribution 4.0 International license. Further distribution of this work must maintain attribution to the author(s) and the published article's title, journal citation, and DOI. polarization switching occurs through a continuous homogeneous mechanism, without formation of new domains of opposite polarization. Homogeneous switching is facilitated in films of nanometer thickness, where inhomogeneous nucleation is strongly suppressed [30].

In order to realize a homogeneous switching, it is necessary that, first, all characteristic times of the process be much shorter than the domain nucleation-growth time and, second, that the sample be a single-domain ferroelectric. Under the ultrafast switching by means of alternating fields, the first condition is easy to accomplish, since the domain nucleation-growth time is sufficiently long, being of order of nanoseconds. In addition, the preparation of single-domain ferroelectrics is a technical problem having several solutions [31-34]. For instance, single-domain states can be made stable by using strain [34] or doping with point defects [35]. Also, there are plenty of ferroelectric films of nanometer thickness, where domain nucleation is suppressed [30].

The homogeneous switching mechanism under the action of an alternating field, involving no nucleation and growth of oppositely polarized domains, can provide reversal times of order of picoseconds. However, the present sources do not provide a sufficiently strong pulse to completely switch the polarization. Experiments [36] have shown that the reversal can be only $40 \%$ of its equilibrium value. Although the reversal is quite fast, occurring in about $10^{-13} \mathrm{~s}$, the reverse polarization rapidly, during the same $10^{-13} \mathrm{~s}$, returns to the initial state, similarly to the dynamics induced by terahertz pulses, when the reversal happens over a picosecond timescale, followed by its fast complete retrieval [37].

Finally, time-dependent density functional theory simulations show that by strongly exciting electrons via laser pulses, it could be possible to change the underlying dynamical potential energy surface, which could result in the polarization switching within tens of picoseconds [38].

In the present paper, we consider the homogeneous way of polarization switching involving alternating fields, but with a rather different setup. The idea of the method is to put a ferroelectric, subject to an external constant electric field, into a resonator cavity. Then the polarization motion induces a resonator feedback field acting back on the ferroelectric. In 
such a way, there is no need to additionally impose external electromagnetic pulses; the ferroelectric produces the required pulse by itself through the feedback field. The polarization switching can be realized in femtoseconds. The suggested method of switching also uses alternating fields, similar to the techniques employing oscillating fields with fixed properties. However, the principal difference is that here the alternating field is not imposed by external sources but is self-organized, being created by moving polarization itself. Such a selforganized feedback field turns out to be essentially more effective than an externally imposed field.

We use the system of units where the Planck constant $\hbar$ is set to one.

\section{EVOLUTION EQUATIONS}

We consider a ferroelectric inserted into a cavity. Generally, if the sample is sufficiently large and especially when it is in contact with other media, say, a dielectric, then it can be separated into domains [39]. However, we consider the case of a cavity containing no other materials inside it except the ferroelectric itself, which is a single-domain sample.

To consider a realistic ferroelectric model, let us take the Hamiltonian in the pseudospin representation [40,41]

$$
\hat{H}=-\Omega \sum_{j} S_{j}^{x}-\frac{1}{2} \sum_{i \neq j} J_{i j} S_{i}^{z} S_{j}^{z}-\sum_{j} \mathbf{E}_{\mathrm{tot}} \cdot \mathbf{P}_{j} .
$$

Here $S_{j}^{\alpha}$ is an $\alpha$ component of the $S=\frac{1}{2}$ spin operator characterizing an electric dipole at site $j, \Omega$ is the tunneling frequency, $J_{i j}=J_{j i}>0$ describes the strength of dipolar interactions, $\mathbf{E}_{\text {tot }}$ is the total electric field acting on dipoles, and

$$
\mathbf{P}_{j}=d_{0} \mathbf{S}_{j}
$$

is a dipolar operator.

This Hamiltonian provides a very good description of the so-called order-disorder ferroelectrics, although it can also be a reasonable approximation for other types of ferroelectrics [40,41]. Among order-disorder ferroelectrics, we mention, for example, $\mathrm{KH}_{2} \mathrm{PO}_{4}, \mathrm{KH}_{2} \mathrm{AsO}_{4}, \mathrm{RbH}_{2} \mathrm{PO}_{4}, \mathrm{RbH}_{2} \mathrm{AsO}_{4}$, $\mathrm{CsH}_{2} \mathrm{PO}_{4}, \mathrm{CsH}_{2} \mathrm{AsO}_{4}, \mathrm{NH}_{4} \mathrm{H}_{2} \mathrm{PO}_{4}$, and $\mathrm{NH}_{4} \mathrm{H}_{2} \mathrm{AsO}_{4}$ and their deuterated analogs, in which $\mathrm{H}_{2}$ is replaced by $\mathrm{D}_{2}$. Similar Hamiltonians also are used to describe relaxor ferroelectrics [42].

The Hamiltonian (1) is the standard, widely used, Hamiltonian for describing macroscopic ferroelectric samples. For finite samples, in general, one should take into account the depolarizing field caused by the charges on the surfaces of the sample [43]. However, there are ways [44] of compensating surface charges, thus reducing or removing depolarizing fields.

Also, considering an external electric field $E_{0}$, applied in the $z$ direction, leads to the appearance of the depolarizing field proportional to $-P_{z}$, which is of order of $\rho d_{0} S$, where $\rho$ is the sample density. The energy, corresponding to the depolarizing field, is $d_{0} P_{z}$, which gives $\rho d_{0}^{2} S$. The latter expression equals the dephasing rate or transverse attenuation defined by $\gamma_{2}=\rho d_{0}^{2} S$. The magnitude of the energy, corresponding to the external field, is $\left|d_{0} E_{0}\right|$, which denotes the dipole rotation frequency defined as $\omega_{0}=\left|d_{0} E_{0}\right|$. For what follows, we will need a sufficiently strong external field such that $\omega_{0}$ is much larger than $\gamma_{2}$. This is necessary to realize the coherent motion of dipoles so that the reversal time is much shorter than the dephasing time. Under the condition $\omega_{0} \gg \gamma_{2}$, corrections, related to the depolarizing field, can be omitted in the Hamiltonian. At the same time, the attenuation rate $\gamma_{2}$ will be taken into account in the equations of motion.

The total electric field consists of two terms

$$
\mathbf{E}_{\mathrm{tot}}=E \mathbf{e}_{x}+E_{0} \mathbf{e}_{z}
$$

where the first term is the field of the resonator cavity, in which the sample is inserted, and the second term is the external constant electric field. The resonator cavity is chosen such that it supports the $\mathrm{TM}_{010}$ fundamental mode, whose electric field is directed along the cavity axis that is taken to be the $x$ axis. The resonator cavity electric field is a feedback field generated by the moving polarization

$$
\mathbf{P}=\frac{d_{0}}{V} \sum_{j}\left\langle\mathbf{S}_{j}\right\rangle
$$

with $V$ the sample volume.

The equation for the feedback field can be derived in the standard way [45]. From the Maxwell equations inside the cavity with an inserted ferroelectric, it is straightforward to get the equation for the electric field

$$
\nabla^{2} \mathbf{E}-\frac{1}{c^{2}} \frac{\partial^{2} \mathbf{E}}{\partial t^{2}}-\frac{4 \pi \sigma}{c^{2}} \frac{\partial \mathbf{E}}{\partial t}=\frac{4 \pi}{c^{2}} \frac{\partial^{2} \mathbf{P}}{\partial t^{2}}
$$

generated by the ferroelectric polarization (4), where $\sigma$ is the conductivity and $c$ is the light velocity. It is possible to look for the solution to this equation in the form

$$
\mathbf{E}(\mathbf{r}, t)=\mathbf{e}(\mathbf{r}) E(t),
$$

where $\mathbf{e}(\mathbf{r})$ is a cavity mode defined by the Helmholtz equation and normalized to the cavity volume $V_{c}$ so that

$$
\frac{1}{V_{c}} \int|\mathbf{e}(\mathbf{r})|^{2} d \mathbf{r}=1 .
$$

We are looking for the $\mathrm{TM}_{010}$ fundamental mode, which, by definition, is the mode directed along the cavity axis, which here is the $x$ axis, which implies the conditions

$$
e_{y}(\mathbf{r})=0, \quad e_{z}(\mathbf{r})=0 .
$$

The mode $x$ component is nonzero inside the cavity, while it satisfies the boundary condition

$$
\left.e_{x}(\mathbf{r})\right|_{r=R}=0
$$

on the cavity cylindrical surface of radius $R$. The expression for the $\mathrm{TM}_{010}$ fundamental mode is known [45] to be presented through the Bessel function of the first kind,

$$
e_{x}(\mathbf{r})=C_{0} J_{0}\left(\frac{\omega}{c} r\right), \quad C_{0}=J_{1}^{-1}\left(\frac{\omega}{c} R\right),
$$

with $C_{0}$ the normalization constant. The boundary condition $e_{x}(R)=0$ corresponds to the first zero of the Bessel function 
$J_{0}(\omega R / c)=0$, which defines the cavity natural frequency

$$
\omega=2.4048 \frac{c}{R} \text {. }
$$

The normalization constant becomes

$$
C_{0}=\frac{1}{J_{1}(2.4048)}=\frac{1}{0.51915}=1.9262 .
$$

Thus the $\mathrm{TM}_{010}$ fundamental mode reads

$$
\mathbf{e}(\mathbf{r})=C_{0} J_{0}\left(\frac{\omega}{c} r\right) \mathbf{e}_{x}=e_{x}(\mathbf{r}) \mathbf{e}_{x},
$$

where $\mathbf{e}_{x}$ is a unit vector along the cavity axis $x$. Then we substitute the expression (6), with the mode (11), into Eq. (5), multiply the latter by the form (11), take into account that

$$
\mathbf{P} \cdot \mathbf{e}(\mathbf{r})=P_{x} e_{x}(\mathbf{r}),
$$

and integrate over the cavity volume. This leads to the equation

$$
\frac{d^{2} E}{d t^{2}}+2 \gamma \frac{d E}{d t}+\omega^{2} E=-4 \pi \eta_{f} \frac{d^{2} P_{x}}{d t^{2}},
$$

in which $\gamma=2 \pi \sigma$ is a cavity attenuation, $\omega$ is the cavity natural frequency (10), and

$$
\eta_{f} \equiv \frac{1}{V_{c}} \int e_{x}(\mathbf{r}) d \mathbf{r}=0.83167
$$

is the filling factor corresponding to the $\mathrm{TM}_{010}$ fundamental mode.

The evolution equations, following from the Heisenberg equations of motion, are

$$
\begin{aligned}
\frac{d S_{i}^{x}}{d t} & =\left(\sum_{j} J_{i j} S_{j}^{z}+d_{0} E_{0}\right) S_{i}^{y}, \\
\frac{d S_{i}^{y}}{d t} & =-\left(\sum_{j} J_{i j} S_{j}^{z}+d_{0} E_{0}\right) S_{i}^{x}+\left(\Omega+d_{0} E\right) S_{i}^{z}, \\
\frac{d S_{i}^{z}}{d t} & =-\left(\Omega+d_{0} E\right) S_{i}^{y} .
\end{aligned}
$$

The observable quantities are given by the statistical averages

$$
s_{\alpha} \equiv \frac{1}{N S} \sum_{j}\left\langle S_{j}^{\alpha}\right\rangle \quad(\alpha=x, y, z),
$$

where $N$ is the number of lattice sites and $S=\frac{1}{2}$ is the spin value.

The attenuation can be taken into account by employing the method of local fields [46,47], where the attenuation is caused by particle interactions acting in the local field formed by other particles, so that the dynamic variables are forced to relax to their local equilibrium values. In the present case, the latter are

$$
\zeta_{\alpha} \equiv \frac{1}{N S} \sum_{j}\left\langle S_{j}^{\alpha}\right\rangle_{\mathrm{loc}},
$$

where $S=\frac{1}{2}$ and the local equilibrium averages are expressed through variables (14) taken at the given moment of time. Since dipolar interactions are of long range, the mean-field approximation is applicable. In this way, the quantity $\left\langle S_{j}^{\alpha}\right\rangle_{\text {loc }}$ is defined as the average

$$
\left\langle S_{j}^{\alpha}\right\rangle_{\mathrm{loc}}=\operatorname{Tr} \hat{\rho}_{\mathrm{loc}} S_{j}^{\alpha},
$$

with the local equilibrium statistical operator

$$
\hat{\rho}_{\text {loc }}=\frac{\exp \left(-\hat{H}_{\mathrm{loc}} / T\right)}{\operatorname{Tr} \exp \left(-\hat{H}_{\mathrm{loc}} T\right)}
$$

for the ensemble of spins with the Hamiltonian

$$
\hat{H}_{\mathrm{loc}}=-\Omega \sum_{j} S_{j}^{x}-\frac{s_{z}}{2} \sum_{i \neq j} J_{i j} S_{j}^{z}-d_{0} E_{0} \sum_{j} S_{j}^{z} .
$$

Accomplishing explicit calculations for the local average (16), we keep in mind low temperatures such that $T \ll J$, where

$$
J \equiv \frac{1}{N} \sum_{i \neq j} J_{i j} .
$$

Thus we obtain the low-temperature local equilibrium values

$$
\begin{gathered}
\zeta_{x}=\frac{\Omega}{\left[\Omega^{2}+\omega_{0}^{2}\left(1-A s_{z}\right)^{2}\right]^{1 / 2}}, \quad \zeta_{y}=0, \\
\zeta_{x}^{2}+\zeta_{z}^{2}=1, \quad \zeta_{z}=-\frac{\omega_{0}\left(1-A s_{z}\right)}{\left[\Omega^{2}+\omega_{0}^{2}\left(1-A s_{z}\right)^{2}\right]^{1 / 2}},
\end{gathered}
$$

where we introduce the notation

$$
A \equiv \frac{J S}{\omega_{0}}
$$

and define the frequency

$$
\omega_{0} \equiv-d_{0} E_{0}>0 .
$$

The positive value of $\omega_{0}$ implies that the external electric field is directed downward.

Thus we come to the mean-field evolution equations for the pseudospin variables

$$
\begin{aligned}
& \frac{d s_{x}}{d t}=-\omega_{0}\left(1-A s_{z}\right) s_{y}-\gamma_{2}\left(s_{x}-\zeta_{x}\right), \\
& \frac{d s_{y}}{d t}=\omega_{0}\left(1-A s_{z}\right) s_{x}+\left(\Omega+\gamma_{2} h\right) s_{z}-\gamma_{2} s_{y}, \\
& \frac{d s_{z}}{d t}=-\left(\Omega+\gamma_{2} h\right) s_{y}-\gamma_{1}\left(s_{z}-\zeta_{z}\right) .
\end{aligned}
$$

Here $\gamma_{1}$ is the longitudinal relaxation rate due to spin-phonon interactions (see $[40,41]$ ), while $\gamma_{2}=\rho d_{0}^{2} S$ is the transverse attenuation caused by dipolar interactions. Usually, $\gamma_{1} \ll \gamma_{2}$.

By introducing the dimensionless feedback field

$$
h \equiv \frac{d_{0} E}{\gamma_{2}} \quad\left(\gamma_{2}=\rho d_{0}^{2} S\right),
$$

where $\rho=N / V$, and taking into account the expression $P_{x}=$ $\rho d_{0} S s_{x}$, we get the feedback-field equation

$$
\frac{d^{2} h}{d t^{2}}+2 \gamma \frac{d h}{d t}+\omega^{2} h=-4 \frac{\gamma_{f}}{\gamma_{2}} \frac{d s_{x}^{2}}{d t^{2}} .
$$

Here

$$
\gamma_{f} \equiv \pi \eta_{f} \rho d_{0}^{2} S=\pi \eta_{f} \gamma_{2}=2.6 \gamma_{2}
$$


is the coupling rate characterizing the interaction between the ferroelectric sample and resonator.

Equations (21) and (23) define the dynamics of the variables $s_{\alpha}$ and the feedback field $h$. The most interesting is the behavior of the dimensionless polarization $s_{z}=s_{z}(t)$ as a function of time for different parameters, under the given initial polarization $s_{z}(0)=s_{0}$.

\section{NUMERICAL SOLUTION}

The polarization switching is realized in the following way. Suppose the ferroelectric sample is initially polarized along the $z$ axis. The sample is placed inside a resonator cavity supporting the $\mathrm{TM}_{010}$ fundamental mode and is subject to an external electric field directed opposite to the initial polarization. The polarization dynamics is governed by Eqs. (21) and (23). In order to precisely describe this dynamics, we have to fix realistic parameters typical for ferroelectrics $[1,2,41]$.

First, let us note that a ferroelectric characterized by the Hamiltonian (1), without the last term containing electric fields, acquires spontaneous polarization below the critical temperature

$$
T_{c}=\Omega\left(\ln \frac{J+2 \Omega}{J-2 \Omega}\right)^{-1} .
$$

This temperature is positive for the tunneling frequency

$$
\Omega<\frac{J}{2} \quad\left(T_{c}>0\right) .
$$

The interaction strength, due to dipolar forces, $J \approx \rho d_{0}^{2}$. The electric dipole is $d_{0}=e_{0} l_{0}$, with $l_{0} \sim 10^{-8} \mathrm{~cm}$ and the electric charge about a proton charge $e_{0}=1.602 \times 10^{-19}$ C. Keeping in mind that one Coulomb is equal to $2.998 \times$ $10^{9} \mathrm{~g}^{1 / 2} \mathrm{~cm}^{3 / 2} \mathrm{~s}^{-1}$, we find $d_{0} \sim 10^{-27} \mathrm{Ccm} \sim 1 \mathrm{D}$, where one Debye is equal to $3.336 \times 10^{-28} \mathrm{Ccm}$. For the density $\rho \sim 10^{22} \mathrm{~cm}^{-3}$, we obtain $\rho d_{0}^{2} \sim 10^{-14} \mathrm{erg}$, that is, $\rho d_{0}^{2} \sim$ $10^{13} \mathrm{~s}^{-1}$. Actually, for typical ferroelectrics $J \sim 10^{2} \mathrm{~K}$, that is, $J \sim 10^{13} \mathrm{~s}^{-1}$. The dipolar forces induce the transverse attenuation $\gamma_{2}=\rho d_{0}^{2} S$. Thus we have

$$
J S \approx \gamma_{2}=\rho d_{0}^{2} S \sim 10^{13} \mathrm{~s}^{-1} .
$$

The longitudinal attenuation, caused by the interaction of pseudospins with phonons, is much smaller than the transverse attenuation, $\gamma_{1} \ll \gamma_{2}$.

The cavity is called resonant, since its natural frequency $\omega$ has to be tuned close to the dipole rotation frequency $\omega_{0}$, satisfying the quasiresonance condition

$$
\left|\frac{\Delta}{\omega}\right| \ll 1 \quad\left(\Delta \equiv \omega-\omega_{0}\right) .
$$

In contrast, the attenuations are to be smaller than $\omega_{0}$, so that

$$
\frac{\gamma_{2}}{\omega_{0}} \ll 1, \quad \frac{\gamma}{\omega} \ll 1
$$

Therefore, for the parameter (19) we get

$$
A \equiv \frac{J S}{\omega_{0}}=\frac{\gamma_{2}}{\omega_{0}} \ll 1
$$

Since $J S=\gamma_{2} \sim 10^{13} \mathrm{~s}^{-1}$, to satisfy the condition (29), we need $\omega_{0}$ to be at least about $10^{14}-10^{15} \mathrm{~s}^{-1}$, which is in

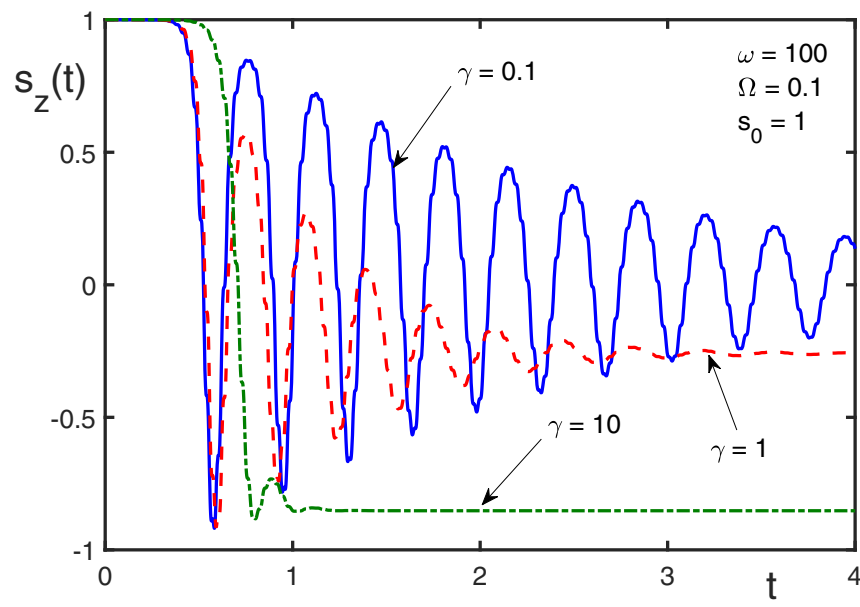

FIG. 1. Polarization switching as a function of time for different values of the resonator attenuation: $\gamma=0.1$ (solid line), $\gamma=1$ (dashed line), and $\gamma=10$ (dash-dotted line). The other parameters are $\Omega=0.1, \omega=100$, and $s_{0}=1$. Here and in the following figures, time is in units of $1 / \gamma_{2}$ and all frequencies are in units of $\gamma_{2}$.

the near infrared or visible light range. This gives the wave vector $k_{0} \equiv \omega_{0} / c$ of order of $\left(3 \times 10^{3}\right)-\left(3 \times 10^{4}\right) \mathrm{cm}^{-1}$ and the wavelength $\lambda \sim 10^{-4}-10^{-3} \mathrm{~cm}$. Resonator cavities in the range of visible light are widespread, and also there exist various cavities operating in the infrared region [48-53].

We solve numerically the system of equations (21) and (23), concentrating our attention on the behavior of the polarization $s_{z}=s(t)$ as a function of time, for different parameters in the admissible range. In the figures, time is measured in units of $1 / \gamma_{2}$ and the frequency parameters are measured in units of $\gamma_{2}$. As initial conditions, we need to fix the values $s_{x}(0)=\sqrt{1-s_{0}^{2}}, s_{y}(0), s_{z}(0) \equiv s(0) \equiv s_{0}, h(0)$, and the time derivative $\dot{h}(0)$. The initial polarization is positive, $s_{0}>0$. If some of other initial conditions, except $s_{0}$, are not zero, the reversal begins immediately at $t=0$. When all of them (except $s_{0}$ ) are zero, there is a time delay. In the figures, we show the results for the initial conditions $s_{y}(0)=0, h(0)=0$, and $\dot{h}(0)=0$, while $s_{0}$ and $s_{x}(0)=\sqrt{1-s_{0}^{2}}$ can be varied. The resonance is assumed when $\omega=\omega_{0}$.

Figure 1 demonstrates the polarization reversal at different values of the resonator attenuation. For small $\gamma$, the polarization oscillates after the switching. Hence, in order to achieve a steady state after the polarization reversal, it is necessary to take larger $\gamma$. For $\gamma=10$, the after-switching oscillations are suppressed. Thus, to avoid oscillations, it is preferable that the resonator ringing time $\tau \equiv 1 / \gamma$ be shorter than the dephasing time $T_{2} \equiv 1 / \gamma_{2}$.

Figure 2 shows the dependence of the polarization switching on the tunneling frequency. The larger the $\Omega$, the shorter the delay time. As is clear from the evolution equations, this is because the tunneling triggers the polarization motion.

In Fig. 3, the role of the frequency $\omega$ is illustrated. The larger the $\omega$, the shorter the delay time and the better the polarization inversion. This happens because the larger frequency makes stronger the coupling between the resonator cavity and the ferroelectric sample. 


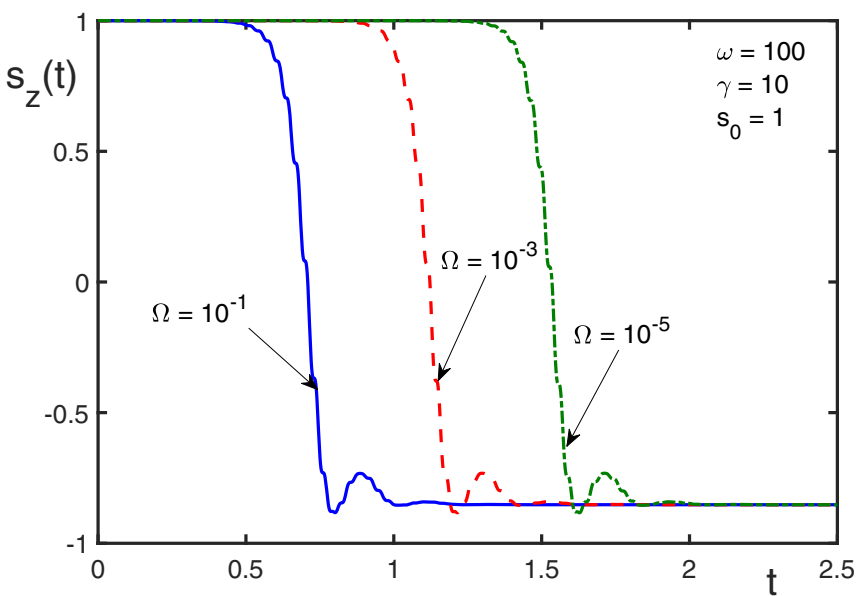

FIG. 2. Polarization switching for varying tunneling frequency: $\Omega=10^{-5}$ (dash-dotted line), $\Omega=10^{-3}$ (dashed line), and $\Omega=10^{-1}$ (solid line). The other parameters are $\omega=100, \gamma=10$, and $s_{0}=1$.

Figure 4 shows a similar dependence of the polarization switching on the frequency $\omega$, as in Fig. 3, but for the initial polarization $s_{0}=0.5$, when $s_{x}(0)=\sqrt{1-s_{0}^{2}}$ is not zero. As is mentioned above, a nonzero $s_{x}(0)$ triggers the start of the polarization motion, so that there is no delay time, and the switching begins from $t=0$.

In Fig. 5, the polarization switching for different initial polarizations is compared: $s_{0}=0.5$ (solid line) and $s_{0}=1$ (dash-dotted line). For $s_{0}=0.5$, the initial transverse component $s_{x}(0)=\sqrt{1-s_{0}^{2}}$ is not zero, because of which the process of switching starts from the very beginning, practically at $t=0$, without delay.

Figure 6 demonstrates that the transverse polarization component $s_{x}$ oscillates around zero. The oscillation is faster for larger $\omega$. The maximal oscillation amplitude corresponds to the moment of the polarization switching.

Since the transverse component $s_{x}$ generates, by means of the relation (23), the dimensionless cavity field $h$, and hence the dimensional electric field inside the cavity $E$, the temporal

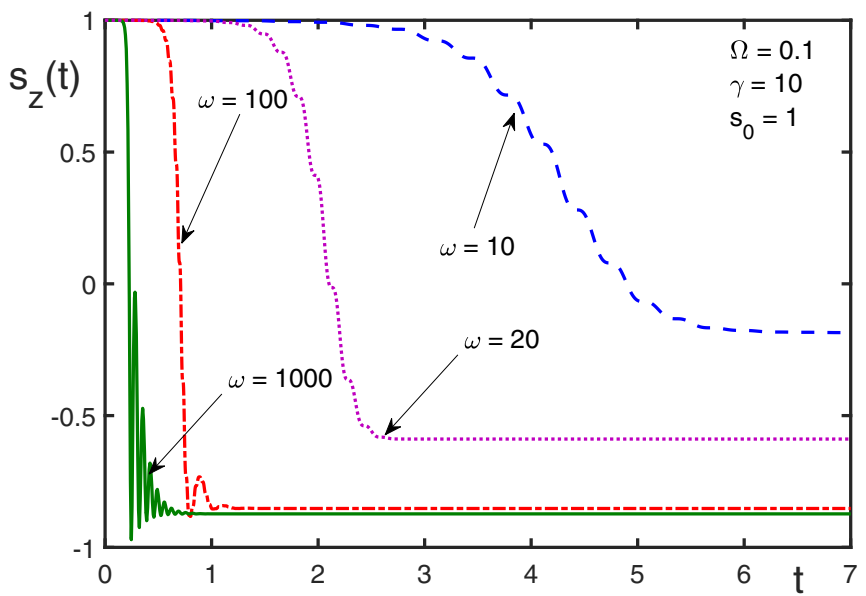

FIG. 3. Dependence of the polarization switching on the frequency: $\omega=10$ (dashed line), $\omega=20$ (dotted line), $\omega=100$ (dashdotted line), and $\omega=1000$ (solid line). The other parameters are $\Omega=0.1, \gamma=10$, and $s_{0}=1$.

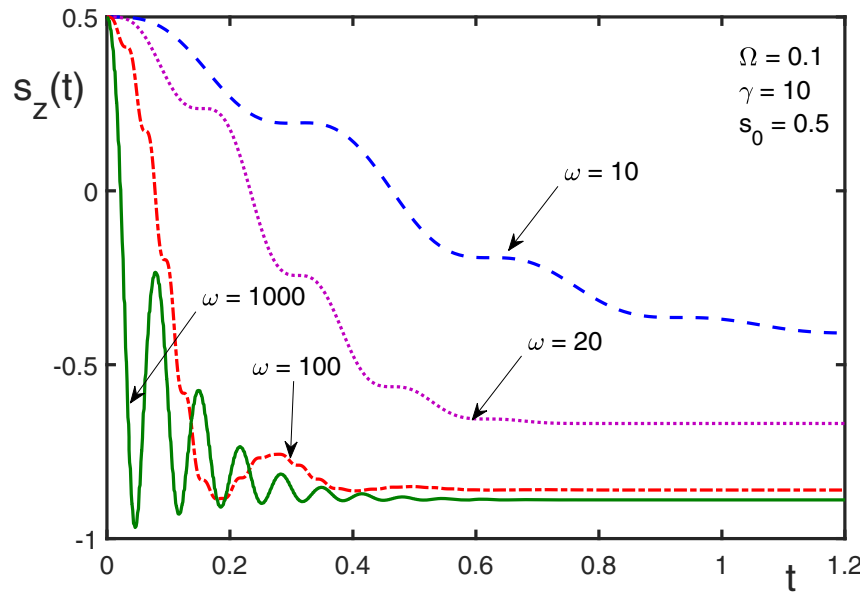

FIG. 4. Dependence of the polarization switching on the varying frequency $\omega$ for the same parameters as in Fig. $3, \Omega=0.1$ and $\gamma=$ 10 , but for the initial polarization $s_{0}=0.5$. Here $\omega=10$ (dashed line), $\omega=20$ (dotted line), $\omega=100$ (dash-dotted line), and $\omega=$ 1000 (solid line).

behavior of $h$, as is seen from Fig. 7, is similar to that of the component $s_{x}$.

\section{ANALYTICAL SOLUTION}

Although the numerical solution of the preceding section gives us an accurate description of the process of polarization switching, it nevertheless is desirable to have analytic solutions that would provide, at least approximately, explicit formulas allowing for a better understanding of the related physics and for straightforward estimates of characteristic quantities. It is convenient to pass to the variables

$$
\begin{aligned}
u & \equiv s_{x}-i s_{y}, \\
w & \equiv|u|^{2}=s_{x}^{2}+s_{y}^{2}, \\
s & \equiv s_{z} .
\end{aligned}
$$

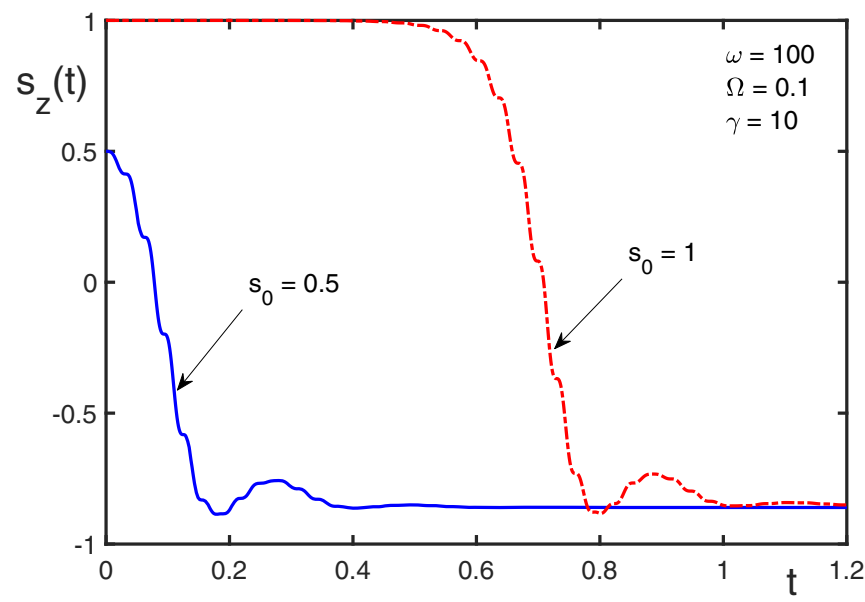

FIG. 5. Polarization switching for different initial polarizations: $s_{0}=0.5$ (solid line) and $s_{0}=1$ (dash-dotted line). The other parameters are $\omega=100, \Omega=0.1$, and $\gamma=10$. For $s_{0}<1$, the delay time is practically absent. 

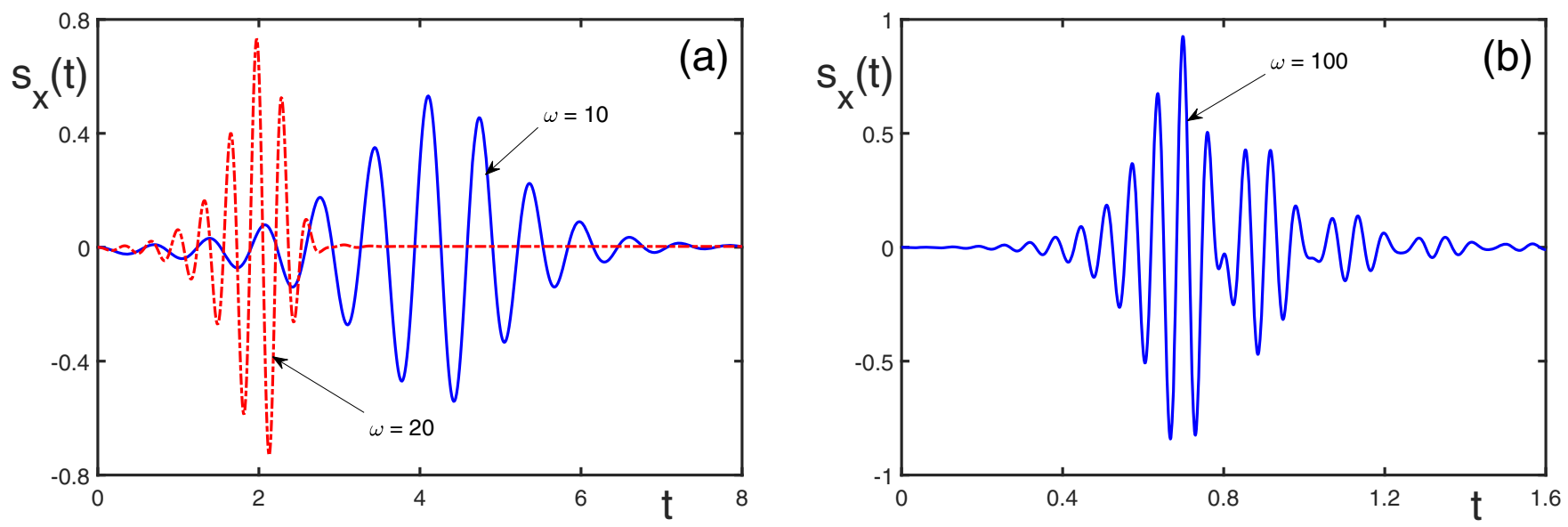

FIG. 6. Temporal dependence of the transverse polarization component $s_{x}$, with the parameters $\Omega=0.1, \gamma=10$, and $s_{0}=1$, for different frequencies: (a) $\omega=10$ (solid line) and $\omega=20$ (dash-dotted line) and (b) $\omega=100$ (solid line).

In terms of these variables, Eqs. (21) transform into the equation for the transverse component

$$
\frac{d u}{d t}=-i \omega_{0}(1-A s) u-\gamma_{2} u-i \gamma_{2}\left(h+\frac{\Omega}{\gamma_{2}}\right) s+\gamma_{2} \zeta_{x},
$$

for the coherence intensity

$$
\frac{d w}{d t}=-2 \gamma_{2} w-i \gamma_{2}\left(h+\frac{\Omega}{\gamma_{2}}\right)\left(u^{*}-u\right) s+\gamma_{2} \zeta_{x}\left(u^{*}+u\right),
$$

and for the polarization

$$
\frac{d s}{d t}=\frac{i}{2} \gamma_{2}\left(h+\frac{\Omega}{\gamma_{2}}\right)\left(u^{*}-u\right)-\gamma_{1}\left(s-\zeta_{z}\right) .
$$

Keeping in mind the case of resonance, defined by Eq. (27), and the existence of the small parameters described in Eqs. (26), (28), and (29), we note that the variables $u$ and $h$ can be classified as fast and the variables $w$ and $s$ as slow. In that case, to solve the given system of equations, it is admissible to resort to the averaging techniques $[54,55]$. Below we follow the variant of the method described in detail in Refs. [56,57]. First, we solve the equations for the fast variables, keeping

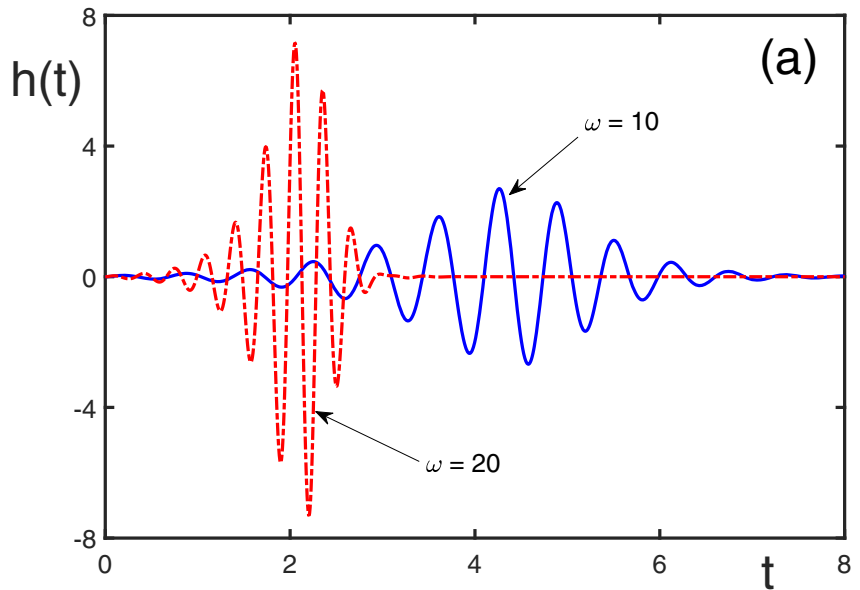

there the slow variables as quasi-integrals of motion. Such a solution is straightforward, although cumbersome, since the equations for the fast variables become linear with respect to the latter, when the slow variables are kept fixed. Then the solutions found for the fast variables are substituted into the equations for the slow variables, with the averaging of the slow-variable equations over time. This yields the equations for the guiding centers, which can be analyzed. All this machinery has been thoroughly described in Refs. [56,57] and its use has been demonstrated in the study of the dynamics of magnetic systems [58-60].

To present the solutions for the fast variables, under fixed slow variables, in a compact form, we take into account the small parameters and introduce various notation. We define the coupling parameter

$$
g \equiv \frac{\gamma_{f} \omega_{0}}{\gamma \gamma_{2}}=2.6 \frac{\omega_{0}}{\gamma},
$$

characterizing the strength of the coupling between the ferroelectric sample and resonator; the coupling function

$$
\alpha \equiv g(1-A s)\left(1-e^{-\gamma t}\right)
$$

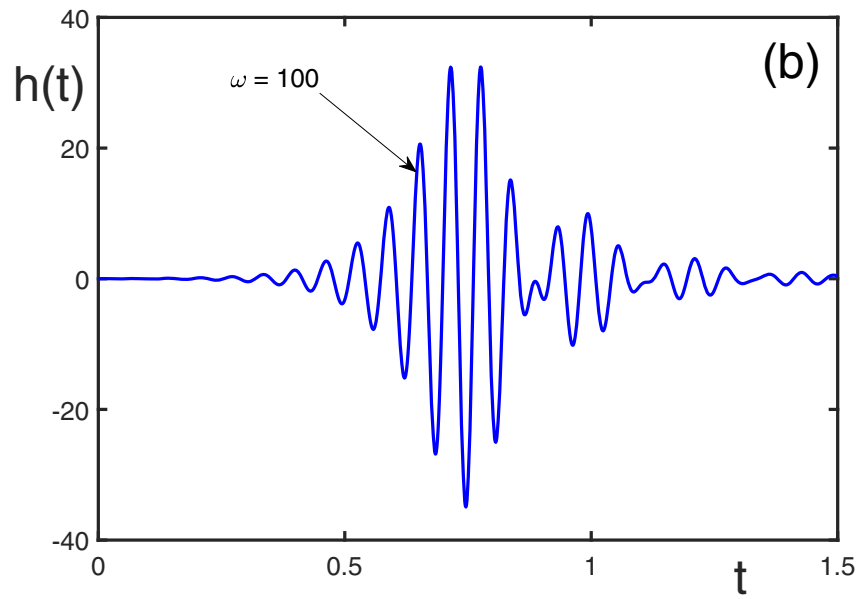

FIG. 7. Dimensionless electric field inside the cavity $h(t)$, as a function of time with the parameters $\Omega=0.1, \gamma=10$, and $s_{0}=1$, for different frequencies: (a) $\omega=10$ (solid line) and $\omega=20$ (dash-dotted line) and (b) $\omega=100$ (solid line). 
describing the dynamics of the ferroelectric-resonator interaction; and the effective frequency

$$
\omega_{\text {eff }} \equiv \omega_{0}(1-A s)-i \gamma_{2}(1-\alpha s) .
$$

Thus we obtain the transverse component

$$
u=\left(u_{0}+\frac{\Omega s+i \gamma_{2} \zeta_{x}}{\omega_{\mathrm{eff}}}\right) \exp \left(-i \omega_{\mathrm{eff}} t\right)-\frac{\Omega s+i \gamma_{2} \zeta_{x}}{\omega_{\mathrm{eff}}}
$$

and the feedback field

$$
h=-i \alpha\left(u^{*}-u\right) .
$$

Note that from Eq. (18) we have

$$
\zeta_{x} \cong \frac{\Omega}{\omega_{0}}, \quad \zeta_{z} \cong-1 .
$$

Substituting the fast variables into the equations for the slow variables and averaging the resulting equations over time gives the guiding-center equations for the coherence intensity,

$$
\frac{d w}{d t}=-2 \gamma_{2}(1-\alpha s) w+2 \gamma_{3}(1-\alpha-\alpha s) s^{2},
$$

and for the polarization

$$
\frac{d s}{d t}=-\gamma_{2} \alpha w-\gamma_{3}(1+s-2 \alpha s)-\gamma_{1}\left(s-\zeta_{z}\right),
$$

where

$$
\gamma_{3} \equiv \gamma_{2} \frac{\Omega^{2}}{\omega_{0}^{2}} .
$$

The parameter $\gamma_{3}$ is very small. However, it cannot be neglected, since it plays an important role in triggering the polarization motion at the initial stage. At the very beginning of the process, when $t \rightarrow 0$, so that

$$
\gamma t \ll 1, \quad \gamma_{1} t \ll 1, \quad \gamma_{2} t \ll 1, \quad \gamma_{3} t \ll 1,
$$

the coupling function (35) is close to zero. Then, keeping in mind that usually $\gamma_{1} \ll \gamma_{2}$, the equations of motion become

$$
\begin{aligned}
\frac{d w}{d t} & =-2 \gamma_{2} w+2 \gamma_{3} s^{2}, \\
\frac{d s}{d t} & =-\gamma_{3}(1+s) \quad(t \rightarrow 0) .
\end{aligned}
$$

Their solutions are

$$
\begin{aligned}
& w \simeq\left(w_{0}-\frac{\gamma_{3}}{\gamma_{2}} s_{0}^{2}\right) e^{-2 \gamma_{2} t}+\frac{\gamma_{3}}{\gamma_{2}} s_{0}^{2}, \\
& s \simeq\left(1+s_{0}\right) e^{-\gamma_{3} t}-1,
\end{aligned}
$$

which, in view of the inequalities (42), can be simplified to

$$
\begin{aligned}
w & \simeq w_{0}+2\left(\gamma_{3} s_{0}^{2}-\gamma_{2} w_{0}\right) t, \\
s & \simeq s_{0}-\gamma_{3}\left(1+s_{0}\right) t,
\end{aligned}
$$

where $w_{0} \equiv w(0)=1-s_{0}^{2}$ and $s_{0} \equiv s(0)$. These are the solutions at the initial stage, when the motion of individual polarizations is not mutually synchronized.

The coupling function (35) grows with time, implying an increase of the magnitude of the resonator feedback field, which collectivizes the individual polarizations, forcing them to move coherently. The influence of the feedback field becomes crucial after the coherence time $t_{\text {coh }}$, when the coupling function grows so that

$$
\alpha s=1 \quad\left(t=t_{\mathrm{coh}}\right) .
$$

This defines the coherence time

$$
t_{\mathrm{coh}}=\tau \ln \frac{g s_{0}\left(1-A s_{0}\right)}{g s_{0}\left(1-A s_{0}\right)-1} \quad\left(\tau \equiv \frac{1}{\gamma}\right),
$$

with $\tau$ the resonator ringing time. If the ferroelectric-resonator coupling is strong, such that $g s_{0} \gg 1$, then the coherence time is

$$
t_{\mathrm{coh}} \simeq \frac{\tau}{g s_{0}\left(1-A s_{0}\right)} .
$$

At the coherence time, the solutions (44), that is,

$$
w_{\mathrm{coh}}=w\left(t_{\mathrm{coh}}\right), \quad s_{\mathrm{coh}}=s\left(t_{\mathrm{coh}}\right),
$$

can be written as

$$
w_{\mathrm{coh}} \simeq w_{0}+2 \gamma_{3} s_{0}^{2} t_{\mathrm{coh}}, \quad s_{\mathrm{coh}} \simeq s_{0},
$$

where we assume that the coupling parameter $g$ is sufficiently large so that inequalities (42) are yet valid at $t_{\text {coh }}$.

After $t_{\text {coh }}$, the coupling function (35) quickly grows, reaching the value $g(1-A s) \simeq g$. At this stage, the parameters $\gamma_{1}$ and $\gamma_{3}$, which are much smaller than $\gamma_{2}$ and especially $g \gamma_{2}$, can be neglected. Then Eqs. (39) and (40) become

$$
\begin{aligned}
& \frac{d w}{d t}=-2 \gamma_{2}(1-g s) w_{0}, \\
& \frac{d s}{d t}=-\gamma_{2} g w \quad\left(t>t_{\mathrm{coh}}\right) .
\end{aligned}
$$

The latter equations enjoy the exact solutions for the coherence intensity

$$
w=\left(\frac{\gamma_{s}}{g \gamma_{2}}\right)^{2} \operatorname{sech}^{2}\left(\frac{t-t_{0}}{\tau_{s}}\right)
$$

and polarization

$$
s=-\frac{\gamma_{s}}{g \gamma_{2}} \tanh \left(\frac{t-t_{0}}{\tau_{s}}\right)+\frac{1}{g} .
$$

The quantities $\gamma_{s}$ and $t_{0}$ are the integration constants that are defined by combining expressions (51) and (52) with Eqs. (49). This gives the switching time $\tau_{s} \equiv 1 / \gamma_{s}$, in which

$$
\gamma_{s}^{2}=\gamma_{g}^{2}+\left(g \gamma_{2}\right)^{2} w_{\text {coh }}, \quad \gamma_{g} \equiv \gamma_{2}\left(g s_{0}-1\right),
$$

and the delay time

$$
t_{0}=t_{\mathrm{coh}}+\frac{\tau_{s}}{2} \ln \left(\frac{\gamma_{s}+\gamma_{g}}{\gamma_{s}-\gamma_{g}}\right) .
$$

The delay time shows the time when the switching starts, while the switching time is the time during which the polarization reversal occurs. The delay time can also be written as

$$
t_{0}=t_{\mathrm{coh}}+\tau_{s} \ln \left(\frac{\gamma_{s}+\gamma_{g}}{g \gamma_{2} \sqrt{w_{\mathrm{coh}}}}\right),
$$

which demonstrates its explicit dependence on $w_{\text {coh }}$. 


\section{CHARACTERISTIC QUANTITIES}

The derived analytic expressions provide a transparent illustration for the role of different system parameters and their combinations. To study more carefully these dependences, let us keep in mind the case of strong ferroelectric-resonator coupling, when $g s_{0} \gg 1$. Then the coherence time (47) takes the form

$$
t_{\mathrm{coh}} \simeq \frac{0.385}{\omega_{0} s_{0}},
$$

which shows that the larger the frequency $\omega_{0}$, the shorter this time.

The quantities (53) read

$$
\gamma_{s} \simeq g \gamma_{2}\left(1+0.385 \frac{\gamma_{2} \Omega^{2}}{\omega_{0}^{3}} s_{0}\right), \quad \gamma_{g} \simeq \gamma_{2} g s_{0} .
$$

The switching time $\tau_{s} \equiv 1 / \gamma_{s}$ becomes

$$
\tau_{s} \simeq 0.385 \frac{\gamma}{\omega_{0} \gamma_{2}},
$$

so a larger $\omega_{0}$ makes the process of switching faster. The delay time (55) acquires the form

$$
t_{0} \simeq t_{\mathrm{coh}}+\tau_{s} \ln \left(\frac{1+s_{0}}{\sqrt{w_{\mathrm{coh}}}}\right),
$$

which depends on the value $w_{\mathrm{coh}}$. The latter is connected with the initial polarization $s_{0}$.

When the system at the initial moment of time is well polarized, with $s_{0}=1$ and $w_{0}=0$,

$$
w_{\mathrm{coh}}=0.77 \frac{\gamma_{2} \Omega^{2}}{\omega_{0}^{3}} \quad\left(s_{0}=1\right) .
$$

In addition, the delay time turns into

$$
t_{0} \simeq \frac{0.385}{\omega_{0}}\left[1+\frac{\gamma}{2 \gamma_{2}} \ln \left(\frac{5.2 \omega_{0}^{3}}{\gamma_{2} \Omega^{2}}\right)\right] \quad\left(s_{0}=1\right) .
$$

In contrast, when $s_{0}=0.5$ and $w_{0}=0.75$, the delay time is

$$
t_{0} \simeq \frac{0.77}{\omega_{0}}\left(1+0.65 \frac{\gamma}{\gamma_{2}}\right) \quad\left(s_{0}=0.5\right) .
$$

To get concrete estimates, let us take the typical values of parameters as have been used when numerically solving the evolution equations: $\omega_{0} \sim 100 \gamma_{2}, \Omega \sim 0.1 \gamma_{2}, \gamma \sim 10 \gamma_{2}$, and $s_{0} \sim 1$. Then $g \sim 10, \gamma_{s} \sim \gamma_{g} \sim 10 \gamma_{2}$, and $\gamma_{3} \sim 10^{-6} \gamma_{2}$. For the coherence time, we have $t_{\mathrm{coh}} \sim 10^{-15} \mathrm{~s}$ and for the switching time we get $\tau_{s} \sim 10^{-14} \mathrm{~s}$. Diminishing $s_{0}$ decreases the delay time. Thus, if $s_{0}=1$ and $w_{0}=0$, then $w_{\text {coh }} \sim 10^{-8}$ and $t_{0} \sim 10^{-13} \mathrm{~s}$; however, if $s_{0} \approx 0.5$, so that $w_{0} \sim 1$, then $t_{0} \sim 10^{-14} \mathrm{~s}$. These estimates are in good agreement with numerical calculations.

An important question is how the switching time is limited in realistic materials, in particular, what the relation is between the switching time $\tau_{s}$ and the cavity ringing time (delay time $\tau \equiv 1 / \gamma$ ). From the above estimates, we find the ratio

$$
\frac{\tau_{s}}{\tau}=0.385 \frac{\gamma^{2}}{\omega_{0} \gamma_{2}} .
$$

It looks like, by varying the system parameters, it is possible to make this ratio rather small. However, there are limitations for the variation of the parameters. Thus, for a good quality cavity one has $\gamma \ll \omega_{0}$. However, $\gamma$ cannot be arbitrarily small, since for $\gamma<\gamma_{2}$ there are oscillations in the polarization. In order to realize a stable switching without oscillations, it is necessary to take $\gamma \gg \gamma_{2}$. Therefore, the ratio (63) lies in the interval

$$
\frac{\gamma_{2}}{\omega_{0}} \ll \frac{\tau_{s}}{\tau} \ll \frac{\omega_{0}}{\gamma_{2}} \quad\left(\gamma_{2} \ll \gamma \ll \omega_{0}\right) .
$$

For the infrared region, where $\omega_{0} / \gamma_{2} \sim 10$, we have

$$
0.1 \ll \frac{\tau_{s}}{\tau} \ll 10 \quad\left(\frac{\omega_{0}}{\gamma_{2}} \sim 10\right),
$$

which actually means that the switching time $\tau_{s}$ is of order of the ringing time $\tau$. In the visible light region, when $\omega_{0} / \gamma_{2} \sim$ 100 , we find

$$
0.01 \ll \frac{\tau_{s}}{\tau} \ll 100 \quad\left(\frac{\omega_{0}}{\gamma_{2}} \sim 100\right) .
$$

This tells us that again the switching time is correlated with the ringing time. For the visible light, it looks admissible to reach the shortest switching time of order $\tau_{s} \sim 10^{-15} \mathrm{~s}$.

\section{COHERENT RADIATION}

The motion of electric dipoles has to produce electromagnetic radiation. If this motion is coherent, the produced radiation should also be coherent. Since the sample is inside a resonator cavity, the radiation can propagate only along the cavity axis, that is, along the $x$ axis. The radiation intensity in the direction of $\mathbf{n}=\mathbf{e}_{x}$ consists of two terms describing incoherent and coherent radiation,

$$
I(\mathbf{n}, t)=I_{\text {inc }}(\mathbf{n}, t)+I_{\text {coh }}(\mathbf{n}, t) .
$$

Radiation produced by moving dipoles can be described in the following way $[45,57,61]$. The incoherent radiation intensity reads

$$
I_{\text {inc }}(\mathbf{n}, t)=\frac{3}{16 \pi} N \omega_{0} \gamma_{0}[1+s(t)]
$$

and the coherent radiation intensity is

$$
I_{\mathrm{coh}}(\mathbf{n}, t)=\frac{3}{32 \pi} N^{2} \omega_{0} \gamma_{0} w(t) F\left(k_{0} \mathbf{n}\right),
$$

with the shape factor

$$
F\left(k_{0} \mathbf{n}\right)=\frac{4}{k_{0}^{2} L^{2}} \sin ^{2}\left(\frac{k_{0} L}{2}\right),
$$

where $L$ is the cavity length and $\gamma_{0}$ is the natural width

$$
\gamma_{0}=\frac{2}{3}\left|\mathbf{d}_{0}\right|^{2} k_{0}^{3} \quad\left(k_{0}=\frac{\omega_{0}}{c}\right) .
$$

For the frequency $\omega_{0} \sim 10^{15} \mathrm{~s}^{-1}$, we have the wavelength $\lambda \sim 10^{-4} \mathrm{~cm}$ and the natural width $\gamma_{0} \sim 10^{4} \mathrm{~s}^{-1}$. Then the radiation intensities at the maximum are

$$
\begin{aligned}
I_{\text {inc }}(\mathbf{n}, t) & \sim N \times 10^{-15} \mathrm{~W}, \\
I_{\text {coh }}(\mathbf{n}, t) & \sim N^{2} F\left(k_{0} \mathbf{n}\right) \times 10^{-16} \mathrm{~W} .
\end{aligned}
$$


The number of dipoles that could radiate coherently can be estimated as $N \sim \rho \lambda^{3}$. If we consider a small sample, with the length $L$ smaller than the radiation wavelength $\lambda$, then the shape factor (68) is of order one. In that case, for the density $\rho \sim 10^{22} \mathrm{~cm}^{-3}$, we get $N \sim 10^{10}$. In addition, for the radiation intensities, we find

$$
I_{\text {inc }}(\mathbf{n}, t) \sim 10^{-5} \mathrm{~W}, \quad I_{\text {coh }}(\mathbf{n}, t) \sim 10^{4} \mathrm{~W} .
$$

When the frequency is $\omega_{0} \sim 10^{14} \mathrm{~s}^{-1}, \lambda \sim 10^{-3} \mathrm{~cm}$ and $\gamma_{0} \sim 10 \mathrm{~s}^{-1}$. The radiation intensities are

$$
\begin{aligned}
I_{\text {inc }}(\mathbf{n}, t) & \sim N \times 10^{-20} \mathrm{~W}, \\
I_{\text {coh }}(\mathbf{n}, t) & \sim N^{2} F\left(k_{0} \mathbf{n}\right) \times 10^{-21} \mathrm{~W} .
\end{aligned}
$$

Considering again coherently radiating dipoles, with the number $N \sim \rho \lambda^{3}$, we have $N \sim 10^{13}$. This gives, for the radiation intensities,

$$
I_{\text {inc }}(\mathbf{n}, t) \sim 10^{-7} \mathrm{~W}, \quad I_{\text {coh }}(\mathbf{n}, t) \sim 10^{5} \mathrm{~W} .
$$

Such a level of radiation can be easily measured. The prevailing coherent component of radiation shows that this radiation is a superradiance.

\section{CONCLUSION}

A method of ultrafast polarization switching in ferroelectrics has been suggested. The main idea is to place a ferroelectric sample into a resonator cavity. In the presence of a constant electric field, directed opposite to the ferroelectric polarization, the sample is in a nonequilibrium state. As soon as the polarization starts moving, it produces an electric field in the cavity. This field acts back on the sample, forcing the polarization to move faster. Thus the ferroelectric itself generates a feedback field accelerating the polarization motion so that it is not necessary to apply external alternating fields, as one usually does to realize polarization switching. It turns out that the self-organized feedback field is essentially more effective for the polarization reversal than an externally imposed field.

The system of equations describing the ferroelectric polarization and feedback field is solved numerically and also analytically by means of averaging techniques. This makes it possible to give a detailed description of the whole procedure, to study the role of the system parameters, and to estimate the characteristic quantities involved in the process. The polarization switching can be realized extremely fast: For the parameters of typical ferroelectrics, the switching time can reach femtoseconds. This ultrafast polarization reversal generates a coherent electromagnetic pulse.
[1] K. Uchino, Ferroelectric Devices (CRC, Boca Raton, 2009).

[2] M. Dawber, K. M. Rabe, and J. F. Scott, Rev. Mod. Phys. 77, 1083 (2005).

[3] C. A. Paz De Araujo, L. D. McMillan, B. M. Melnick, J. D. Cuchiaro, and J. F. Scott, Ferroelectrics 104, 241 (1990).

[4] A. Chanthbouala, V. Garcia, R. O. Cherifi, K. Bouzehouane, S. Fusil, X. Moya, S. Xavier, H. Yamada, C. Deranlot, N. D. Mathur, M. Bibes, A. Barthelemy, and J. Grollier, Nat. Mater. 11, 860 (2012).

[5] S. H. Jo, T. Chang, I. Ebong, B. B. Bhadviya, P. Mazumder, and W. Lu, Nano Lett. 10, 1297 (2010).

[6] M. K. Kim and J. S. Lee, Nano Lett. 19, 2044 (2019).

[7] X. Zhou, J. Jankowska, H. Dong, and O. V. Prezhdo, J. Energy Chem. 27, 637 (2018).

[8] W. Li, Y. Y. Sun, L. Li, Z. Zhou, J. Tang, and O. V. Prezhdo, J. Am. Chem. Soc. 140, 15753 (2018).

[9] A. N. Kolmogorov, Izv. Acad. Nauk. Math. 3, 355 (1937).

[10] M. Avrami, J. Chem. Phys. 8, 212 (1940).

[11] W. J. Merz, Phys. Rev. 95, 690 (1954).

[12] Y. Ishibashi and Y. Takagi, J. Phys. Soc. Jpn. 31, 506 (1971).

[13] A. K. Tagantsev, L. E. Cross, and J. Fousek, Domains in Ferroic Crystals and Thin Films (Springer, New York, 2010).

[14] P. K. Larsen, G. L. M. Kampschöer, M. J. E. Ulenaers, G. A. C. M. Spierings, and R. Cuppens, Appl. Phys. Lett. 59, 611 (1991).

[15] R. Xu, S. Liu, I. Grinberg, J. Karthik, A. R. Damodaran, A. M. Rappe, and L. W. Martin, Nat. Mater. 14, 79 (2015).

[16] H. Lu, C. W. Bark, D. Esque de los Ojos, J. Alcala, C. B. Eom, G. Catalan, and A. Gruverman, Science 336, 59 (2012).
[17] R. V. Wang, D. D. Fong, F. Jiang, M. J. Highland, P. H. Fuoss, C. Thompson, A. M. Kolpak, J. A. Eastman, S. K. Streiffer, A. M. Rappe, and G. B. Stephenson, Phys. Rev. Lett. 102, 047601 (2009).

[18] S. Fahy and R. Merlin, Phys. Rev. Lett. 73, 1122 (1994).

[19] J. B. Ciaran and K. A. Nelson, J. Chem. Phys. 107, 9691 (1997).

[20] J. Li, B. Nagaraj, H. Liang, W. Cao, C. H. Lee, and R. Ramesh, Appl. Phys. Lett. 84, 1174 (2004).

[21] K. Istomin, V. Kotaidis, A. Plech, and Q. Kong, Appl. Phys. Lett. 90, 022905 (2007).

[22] K. Takahashi, N. Kida, and M. Tonouchi, Phys. Rev. Lett. 96, 117402 (2006).

[23] A. Cavalleri, S. Wall, C. Simpson, E. Statz, D. W. Ward, K. A. Nelson, M. Rini, and R. W. Schoenlein, Nature (London) 442, 664 (2006).

[24] T. Qi, Y. H. Shin, K. L. Yeh, K. A. Nelson, and A. M. Rappe, Phys. Rev. Lett. 102, 247603 (2009).

[25] I. Katayama, H. Aoki, J. Takeda, H. Shimosato, M. Ashida, R. Kinjo, I. Kawayama, M. Tonouchi, M. Nagai, and K. Tanaka, Phys. Rev. Lett. 108, 097401 (2012).

[26] F. Chen, Y. Zhu, S. Liu, Y. Qi, H. Y. Hwang, N. C. Brandt, J. Lu, F. Quirin, H. Enquist, P. Zalden et al., Phys. Rev. B 94, 180104(R) (2016).

[27] C. Hauf, A. A. H. Salvador, M. Holtz, M. Woerner, and T. Elsaesser, Struct. Dyn. 5, 024501 (2018).

[28] A. Von Hoegen, R. Mankowsky, M. Fechner, M. Forst, and A. Cavalleri, Nature (London) 555, 79 (2018).

[29] A. Subedi, Phys. Rev. B 92, 214303 (2015).

[30] M. J. Highland, T. T. Fister, M.-I. Richard, D. D. Fong, P. H. Fuoss, C. Thompson, J. A. Eastman, S. K. Streiffer, and G. B. Stephenson, Phys. Rev. Lett. 105, 167601 (2010). 
[31] A. M. Bratkovsky and A. P. Levanyuk, Appl. Phys. Lett. 89, 253108 (2006).

[32] P. Aguado-Puente and J. Junquera, Phys. Rev. Lett. 100, 177601 (2008).

[33] A. M. Bratkovsky and A. P. Levanyuk, J. Comput. Theor. Nanosci. 6, 465 (2009).

[34] N. A. Pertsev and H. Kohlstedt, Phys. Rev. Lett. 98, 257603 (2007).

[35] L. Zhang and X. Ren, Phys. Rev. B 73, 094121 (2006).

[36] R. Mankowsky, A. von Hoegen, M. Först, and A. Cavalleri, Phys. Rev. Lett. 118, 197601 (2017).

[37] D. S. Rana, I. Kawayama, K. Mavani, K. Takahashi, H. Murakami, and M. Tonouchi, Adv. Mater. 21, 2881 (2009).

[38] C. Lian, Z. A. Ali, H. Kwon, and B. M. Wong, J. Phys. Chem. Lett. 10, 3402 (2019).

[39] A. P. Levanyuk and I. B. Misirlioglu, J. Appl. Phys. 119, 024109 (2009).

[40] R. Blinc and B. Zeks, Adv. Phys. 21, 693 (1972).

[41] R. Blinc and B. Zeks, Soft Modes in Ferroelectrics and Antiferroelectrics (North-Holland, Amsterdam, 1974).

[42] A. A. Bokov and Z. G. Ye, J. Mater. Sci. 41, 31 (2006).

[43] Y. Watanabe, Solid State Phen. 189, 57 (2012).

[44] Physics of Ferroelectrics, edited by K. M. Rabe, C. H. Ahn, and J. M. Triscone (Springer, Berlin, 2007).

[45] L. Mandel and E. Wolf, Optical Coherence and Quantum Optics (Cambridge University Press, Cambridge, 1995).

[46] R. K. Wangsness, Phys. Rev. 98, 927 (1955).

[47] V. I. Yukalov and E. P. Yukalova, Phys. Rev. A 78, 063610 (2008).
[48] M. Garin, R. Fenollosa, R. Alcubilla, L. Shi, L. F. Marsal, and F. Meseguer, Nat. Commun. 5, 3440 (2014).

[49] C. Lecaplain, C. Javerzac-Galy, M. L. Gorodetsky, and T. J. Kippenberg, Nat. Commun. 7, 13383 (2016).

[50] A. Osman, M. Nedeljkovic, J. Soler Penades, Y. Wu, Z. Qu, A. Z. Khokhar, K. Debnath, and G. Z. Mashanovich, Opt. Lett. 43, 5997 (2018).

[51] S. Radosavljevic, N. T. Beneitez, A. Katumba, M. Muneeb, M. Vanslembrouck, B. Kuyken, and G. Roelkens, Opt. Mater. Express 8, 824 (2018).

[52] K. Yao and Y. Liu, ACS Photon. 5, 844 (2018).

[53] T. H. Xiao, Z. Zhao, W. Zhou, C. Y. Chang, S. Y. Set, M. Takenaka, H. K. Tsang, Z. Cheng, and K. Goda, Opt. Lett. 43, 2885 (2018).

[54] N. N. Bogolubov and Y. A. Mitropolsky, Asymptotic Methods in the Theory of Nonlinear Oscillations (Gordon \& Breach, New York, 1961).

[55] V. Burd, Method of Averaging for Differential Equations on an Infinite Interval (Chapman and Hall, Boca Raton, 2007).

[56] V. I. Yukalov, Phys. Rev. B 53, 9232 (1996).

[57] V. I. Yukalov and E. P. Yukalova, Phys. Part. Nucl. 31, 561 (2000).

[58] V. I. Yukalov, M. G. Cottam, and M. R. Singh, Phys. Rev. B 60, 1227 (1999).

[59] V. I. Yukalov, Phys. Rev. B 71, 184432 (2005).

[60] V. I. Yukalov and E. P. Yukalova, Phys. Rev. B 98, 144438 (2018).

[61] M. Gross and S. Haroche, Phys. Rep. 93, 301 (1982). 